\title{
COMPORTAMENTO BALÍSTICO DO COMPOSTO CERÂMICO $\mathrm{Al}_{2} \mathrm{O}_{3}-\mathrm{Nb}_{2} \mathrm{O}_{5}-\mathrm{LiF}^{*}$
}

\author{
Jheison Lopes dos Santos ${ }^{1}$ \\ Rubens Lincoln Santana Blazutti Marçal ${ }^{2}$ \\ Sérgio Neves Monteiro ${ }^{3}$ \\ Daniel Navarro da Rocha ${ }^{4}$ \\ Alaelson Vieira Gomes 5 \\ Édio Pereira Lima Jr ${ }^{4}$ \\ Luís Henrique Leme Louro ${ }^{5}$
}

Resumo

Sistemas de blindagens multicamada têm sido estudadas pela sua ótima capacidade de proteção, devido à combinação de diferentes materiais que constituem seus componentes. A primeira camada é constituída por um componente cerâmico, cujo objetivo principal é fragmentar o projétil, dissipando grande parte de sua energia. $A$ camada intermediária compreende tecido de aramida ou matriz polimérica com fibras naturais, que também dissipa energia tanto do projétil quanto do cerâmico e opera retendo os fragmentos cerâmicos. Neste trabalho, pela primeira vez, o sistema cerâmico $\mathrm{Al}_{2} \mathrm{O}_{3}-\mathrm{Nb}_{2} \mathrm{O}_{5}$ - LiF tem seu comportamento dinâmico avaliado balisticamente. Este composto cerâmico foi submetido a impactos balísticos de projéteis $7,62 \mathrm{~mm}$, para testar este cerâmico do primeiro componente de um sistema de blindagens multicamadas. Os resultados obtidos mostraram que a adição de LiF ao composto cerâmico $\mathrm{Al}_{2} \mathrm{O}_{3}-4 \%$ p. $\mathrm{Nb}_{2} \mathrm{O}_{5}$, sinterizado a $1.300{ }^{\circ} \mathrm{C}$, obteve desempenho comparável ao obtido por $\mathrm{Al}_{2} \mathrm{O}_{3}-4 \%$ p. $\mathrm{Nb}_{2} \mathrm{O}_{5}$ sem $\mathrm{LiF}$, sinterizado a $1.400{ }^{\circ} \mathrm{C}$. Os resultados revelaram que, ao serem submetidos a esses impactos balísticos, apresentaram valores comparáveis aos esperados de pressão e de velocidade de partícula na interface de impacto projétil-cerâmico. Dessa forma, a adição de fluoreto de lítio ao composto cerâmico alumina-nióbia possibilitou a redução em $100{ }^{\circ} \mathrm{C}$ na temperatura de sinterização, sem comprometer o desempenho balístico e exibindo parâmetros de choque comparáveis, e redução de custo.

Palavras-chave: $\mathrm{Al}_{2} \mathrm{O}_{3}$; $\mathrm{LiF} ; \mathrm{Nb}_{2} \mathrm{O}_{5}$; Blindagem balística; Comportamento Dinâmico.

\begin{abstract}
BALLISTIC BEHAVIOR OF $\mathrm{AL}_{2} \mathrm{O}_{3}-\mathrm{NB}_{2} \mathrm{O}_{5}$-LIF CERAMIC COMPOUND Abstract

Multilayered armor systems have been studied for their optimal protection capacity, due to the combination of different materials that make up its components. The first layer consists of a ceramic component, whose the main purpose if to fragment the projectile, dissipating much of its energy. The intermediate layer comprises aramid fabric or polymeric matrix with natural fibers, which also dissipates energy from the projectile and operates retaining ceramic fragments. In this work, for the first time, $\mathrm{Al}_{2} \mathrm{O}_{3}-\mathrm{Nb}_{2} \mathrm{O}_{5}-\mathrm{LiF}$ ceramic system has its dynamic behavior evaluated ballistically. This ceramic composite was subjected to ballistic impacts of $7.62 \mathrm{~mm}$ projectile, to test this ceramic as the first component of a multilayered armor system. The results showed that the addition of $\mathrm{LiF}$ to the $\mathrm{Al}_{2} \mathrm{O}_{3}-4 \mathrm{wt} \% \mathrm{Nb}_{2} \mathrm{O}_{5}$ ceramic compound, sintered at 1,300 ${ }^{\circ} \mathrm{C}$, achieved a performance comparable to those obtained by $\mathrm{Al}_{2} \mathrm{O}_{3}-4 \mathrm{wt} \% \mathrm{Nb}_{2} \mathrm{O}_{5}$ without LiF, sintered at $1,400{ }^{\circ} \mathrm{C}$. The results showed that, when subjected to ballistic impacts, they presented comparable values to those expected for pressure and particle velocity at the projectile-ceramic impact interface. Thus, the lithium fluoride addition to the alumina-niobia ceramic compound allowed the reduction at $100{ }^{\circ} \mathrm{C}$ in the sintering temperature, without compromising ballistic performance and exhibiting comparable shock parameters, and cost reduction.
\end{abstract}

Keywords: $\mathrm{Al}_{2} \mathrm{O}_{3}$; LiF; $\mathrm{Nb}_{2} \mathrm{O}_{5}$; Ballistic armor; Dynamic behavior 
1 Doutor em Ciência dos Materiais, Pós-Doutorando, Seção de Engenharia Mecânica e de Materiais, Instituto Militar de Engenharia, Rio de Janeiro, RJ, Brasil.

2 Doutor em Ciência dos Materiais, Pós-Doutorando, Seção de Engenharia Mecânica e de Materiais, Instituto Militar de Engenharia, Rio de Janeiro, RJ, Brasil.

3 Ph.D. em Engenharia Metalúrgica e de Materiais, Professor, Seção de Engenharia Mecânica e de Materiais, Instituto Militar de Engenharia, Rio de Janeiro, RJ, Brasil.

4 Doutor em Ciência dos Materiais, Pós-Doutorando, Seção de Engenharia Mecânica e de Materiais, Instituto Militar de Engenharia, Rio de Janeiro, RJ, Brasil.

5 Doutor em Ciência dos Materiais, Professor, Seção de Engenharia Mecânica e de Materiais, Instituto Militar de Engenharia, Rio de Janeiro, RJ, Brasil.

6 Mestre em Ciência dos Materiais, Professor, Seção de Engenharia Mecânica e de Materiais, Instituto Militar de Engenharia, Rio de Janeiro, RJ, Brasil.

7 Ph.D. em Engenharia Metalúrgica e de Materiais, Professor, Seção de Engenharia Mecânica e de Materiais, Instituto Militar de Engenharia, Rio de Janeiro, RJ, Brasil. 


\section{INTRODUÇÃO}

Vários esforços vêm sendo realizados visando o desenvolvimento de sistemas leves de blindagem. Portanto, cerâmicas avançadas, polímeros e compósitos de matriz cerâmica ou de matriz polimérica têm sido estudados, a fim de proporcionar blindagens de baixo custo com desempenho otimizado.

Geralmente, os sistemas de blindagem mais eficientes são compostos por camadas de diferentes materiais. Isso explora as diferentes propriedades de cada um desses materiais, ocasionando o efeito sinergético e aumentando o desempenho da blindagem. Assim, na blindagem multicamada, as propriedades dos diferentes materiais são complementares para evitar a penetração do projétil e preservar a integridade do alvo [1,2].

Devido a sua alta resistência à compressão e dureza, muitos materiais cerâmicos são usados como o primeiro componente em um sistema de blindagem multicamadas. Sua principal função é dissipar a maior parte da energia do projétil, através da erosão e fragmentação de sua ponta, e como efeito há a fragmentação da camada cerâmica [3-10]. A segunda camada, logo atrás da cerâmica, pode ser uma fibra polimérica de elevada resistência, tal como aramida, ou poliéster de ultra alto peso molecular, composta por compósitos de matriz polimérica e fibras. A função desta camada intermediária é reter os fragmentos gerados da primeira camada de material frágil, bem como ajudar na dissipação da energia do projétil.

Visando o aprimoramento do componente cerâmico, principalmente à base de alumina, Gomes [4] buscou reduzir a temperatura de sinterização da alumina. Para isto, adicionou diferentes frações de nióbia à alumina, utilizando diferentes temperaturas de sinterização. Seus resultados indicaram que a adição de $4 \%$ de nióbia na alumina possibilitou a redução da temperatura de sinterização deste cerâmico de $1600^{\circ} \mathrm{C}$ para $1450^{\circ} \mathrm{C}$, mantendo sem perda na capacidade protetiva. Posteriormente, Jesus [11] aditivou o composto cerâmico $\mathrm{Al}_{2} \mathrm{O}_{3}-4 \% \mathrm{Nb}_{2} \mathrm{O}_{5} \mathrm{com} \mathrm{LiF}$ para reduzir ainda mais esta temperatura de sinterização. Utilizou frações de $1 \%, 2 \%$ e $3 \%$ em peso de fluoreto de lítio. Foi observado que a adição de $1 \%$ permitiu uma boa densificação (da ordem de $90 \%$ da densidade teórica) na temperatura de sinterização de $1350^{\circ} \mathrm{C}$. Santos [12], otimizou a adição de LiF em $0,5 \%$ em peso, obtendo cerca de $90 \%$ da densificação teórica a $1300^{\circ} \mathrm{C}$.

Recentemente, esforços de pesquisa têm sido feitos utilizando fibras naturais em matrizes poliméricas. Foram estudadas fibras de cana-de-açúcar, de curauá, de juta, de sisal, de bambu, e de rami [13-18]. Todas as fibras analisadas apresentaram um bom desempenho como componente intermediário de um sistema de blindagem multicamada, sendo comparáveis ao desempenho de aramida.

Para altas taxas de carregamento, o fenômeno de deformação ocorre mais localizado, ou seja, existem regiões do corpo que ainda não sentiram a influência da passagem das ondas de tensão e deformação. Esta perturbação localizada, causada pelo carregamento dinâmico, propaga-se no sólido sob a forma de uma onda de tensão. Neste processo, as tensões se movem através do material com uma frente que delimita uma região deformada de uma região não deformada. A transmissão interna da tensão átomo a átomo move-se através do corpo a velocidades específicas, o que pode ser determinado com boa aproximação.

O comportamento balístico dos materiais está ligado à sua resposta dinâmica a altas taxas de deformação. Portanto, a solicitação externa imposta pode produzir ondas elásticas, plásticas ou de choque como resposta [19]. A onda elástica é aquela que transmite tensões menores do que a tensão de escoamento do material, ainda em seu 
regime elástico. A velocidade da onda elástica $\left(\mathrm{C}_{0}\right)$ em um meio contínuo depende do módulo de elasticidade $(E)$ e da densidade desse material $(\rho)$, de acordo com a Equação 1.

$$
C_{0}=\sqrt{\frac{E}{\rho}}
$$

As ondas plásticas possuem menor velocidade de propagação, e são produzidas após o valor da tensão ultrapassar o limite de escoamento do material. Nos casos em que o limite de escoamento dinâmico do material é amplamente excedido pelas ondas de tensão, que possuem frentes íngremes, as tensões de cisalhamento podem ser negligenciadas. Isso é possível quando o material se comporta como se fosse um fluido e pode ser modelado com base na equação do gás ideal. Estas ondas são causadas por taxas de deformação intermédias e altas $\left(\mathbf{1 0}^{\mathbf{2}} \boldsymbol{s}^{-\mathbf{1}}<\dot{\boldsymbol{\varepsilon}}<\mathbf{1 0}^{\mathbf{4}} \boldsymbol{s}^{\mathbf{- 1}}\right)$, e são chamadas ondas de choque [19]. O fenômeno de deformação dinâmica em altas taxas é governado pelas equações de conservação propostas por Rankine-Hugoniot [20,21]. As Equações 2, 3 e 4 representam, respectivamente, a conservação da massa, do momento e da energia.

$$
\begin{array}{r}
\rho_{0} U_{s}=\rho\left(U_{s}-U_{P}\right) \\
\left(P-P_{0}\right)=\rho_{0} U_{S} U_{P} \\
E-E_{0}=\frac{\left(\mathbf{P}+\mathbf{P}_{0}\right)\left(\mathbf{V}_{0}-\mathbf{V}\right)}{2}
\end{array}
$$

O presente trabalho apresenta pela primeira vez os valores de propriedades elásticas do composto cerâmico $\mathrm{Al}_{2} \mathrm{O}_{3}-\mathrm{Nb}_{2} \mathrm{O}_{5}-\mathrm{LiF}$, com composição otimizada por Santos [12]. Além disso, este novo componente cerâmico foi ensaiado balisticamente contra munição 7,62 mm, e são apresentados os valores de pressão e de velocidade de partícula na interface projétil-cerâmico, mediante o impacto balístico.

\section{MATERIAIS E MÉTODOS}

\subsection{Processamento cerâmico}

Os pós de alumina (Treibacher Scheifmittel) e dos aditivos de sinterização niobia (CBMM) e fluoreto de lítio (Vetec) utilizados neste trabalho foram de elevada pureza (superiores a 98,5\%). O ligante orgânico polietileno glicol (PEG) 400 (Vetec) foi utilizado para conferir maior resistência mecânica do corpo cerâmico a verde. A etapa de moagem do processamento cerâmico foi implementada com a adição de água ultrapura à mistura de cerâmica $(94,53 \%$ de alumina e 3,94\%de nióbia) e do ligante orgânico (1,53\%), utilizando bolas de diferentes tamanhos, de alumina, como elemento de moagem. Este processo de cominuição e homogeneização da mistura durou 8 horas. Depois disso, o pó úmido foi seco a $70{ }^{\circ} \mathrm{C}$ durante cerca de $48 \mathrm{~h}$. A mistura seca foi macerada e desaglomerada em almofariz cerâmico e pistilo, e depois peneirada através de uma de abertura de 0,255 mm. Após a preparação da mistura inicial de referência, adicionou-se $0,5 \%$ em peso de LiF definido anteriormente. Para isso, foram reproduzidos os mesmos passos de processamento executados para obter a mistura de referência inicial.

Para a conformação mecânica, utilizou-se a prensagem uniaxial a frio com matriz flutuante, com tensão de compactação de $50 \mathrm{MPa}$ em duas matrizes metálicas distintas: uma matriz circular de $57 \mathrm{~mm}$ de diâmetro interno e $60 \mathrm{~g}$ de material, e outra matriz de seção reta hexagonal, usando $100 \mathrm{~g}$ de massa. A matriz circular foi utilizada 
para confeccionar os corpos de prova para o ensaio de velocidade residual (Figura 1(a)), enquanto a matriz hexagonal foi utilizada na confecção dos corpos de prova para o ensaio balístico seguido de indentação na plastilina, como blindagem multicamada (Figura 1(b)).

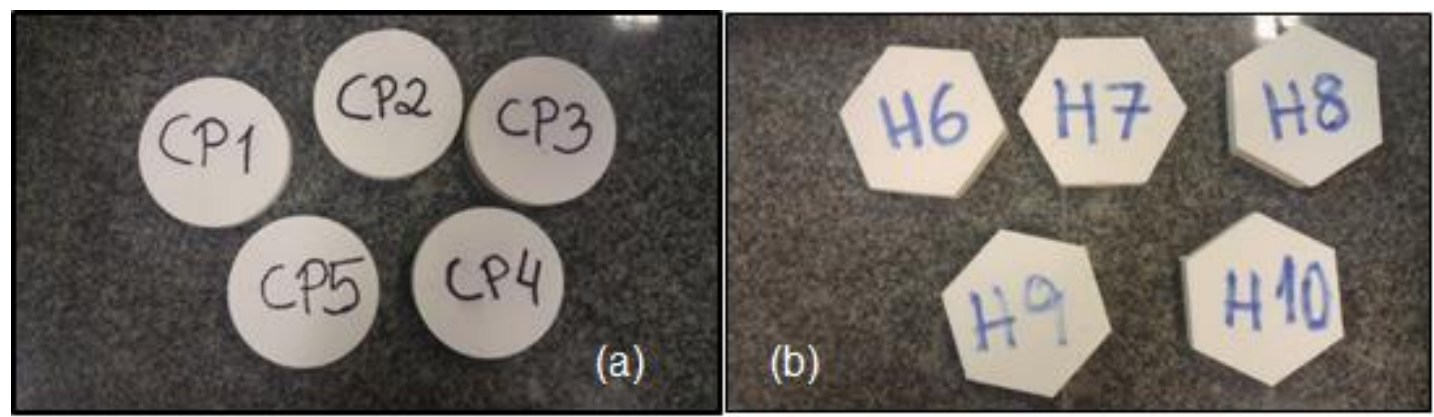

Figura 1. Corpos de prova (a) cilíndricos utilizados no ensaio de velocidade residual, e (b) hexagonais utilizados no ensaio de indentação na plastilina em blindagem multicamada.

A sinterização das amostras foi realizada num forno modelo FE-1700, da marca INTI, com o ciclo térmico adotado na sinterização descrito a seguir:

1) Aquecimento de $25^{\circ} \mathrm{C}$ a $158^{\circ} \mathrm{C}$ à razão de $1^{\circ} \mathrm{C} / \mathrm{min}$;

2) Patamar de $158^{\circ} \mathrm{C}$ durante $1 \mathrm{~h}$;

3) Aquecimento de $158^{\circ} \mathrm{C}$ a $375^{\circ} \mathrm{C}$ à razão de $1^{\circ} \mathrm{C} / \mathrm{min}$;

4) Aquecimento de $375^{\circ} \mathrm{C}$ a $1000^{\circ} \mathrm{C}$ à razão de $8^{\circ} \mathrm{C} / \mathrm{min}$;

5) Patamar de $1000^{\circ} \mathrm{C}$ durante $1 \mathrm{~h}$;

6) Aquecimento a partir de $1000^{\circ} \mathrm{C}$ até à temperatura final de sinterização $\left(1300{ }^{\circ} \mathrm{C}\right.$, $1350^{\circ} \mathrm{C}$, ou $1400^{\circ} \mathrm{C}$ ) à razão de $5^{\circ} \mathrm{C} / \mathrm{min}$;

7) Patamar de sinterização à temperatura de sinterização desejada $\left(1300{ }^{\circ} \mathrm{C}, 1350\right.$ ${ }^{\circ} \mathrm{C}$, ou $1400^{\circ} \mathrm{C}$ ) durante $3 \mathrm{~h}$.

8) Arrefecimento a $3^{\circ} \mathrm{C} / \mathrm{min}$ até $700^{\circ} \mathrm{C}$, quando o forno foi desligado e arrefecido por sua própria inércia.

\subsection{Caracterizações}

Pela primeira vez, uma composição cerâmica à base de alumina-nióbia-fluoreto de lítio foi avaliada dinamicamente por ensaio balístico. Os testes balísticos foram realizados no Centro de Avaliação do Exército Brasileiro (CAEx), utilizando munição de $7,62 \times 51 \mathrm{~mm}$, de $9,7 \mathrm{~g}$. Os alvos cerâmicos foram posicionados a $15 \mathrm{~m}$ do dispositivo de disparo, de acordo com a norma ABNT NBR 15000 [22]. Foram realizados dois ensaios balísticos: o primeiro de velocidade residual, e o segundo de indentação na plastilina.

\subsubsection{Ensaio balístico de velocidade residual}

Neste ensaio, através de um radar Doppler da marca Weibel, modelo SL-520P, foi possível aferir as velocidades do projétil antes e após o impacto. Dessa forma, mediuse a energia absorvida pelo componente cerâmico da blindagem pela variação de energia cinética do projétil. Os corpos de prova (Figura 1(a)) foram fixados diretamente no suporte. Tal procedimento foi adotado para avaliar o componente cerâmico na condição mais crítica, sem qualquer interferência de outros componentes da blindagem multicamada. 


\subsubsection{Ensaio balístico de penetração na plastilina}

Neste segundo teste, uma única camada de aramida foi inserida à frente do cerâmico para auxiliar na contenção de fragmentos, evitando danos aos equipamentos (Figura 2(a)). Após o corpo cerâmico, duas monocamadas e duas placas de aramida com 4 $\mathrm{mm}$ de espessura cada foram incorporadas, totalizando aproximadamente $10 \mathrm{~mm}$ de espessura. Após estas camadas, foi inserida uma placa de alumínio 5054, de 12 × 15 $\mathrm{cm}$ e $5 \mathrm{~mm}$ de espessura. Todas as camadas foram coladas com adesivo polimérico à base de poliuretano (Figura 2(b)). A fixação dessa blindagem multicamada foi feita em bloco de plastilina, mimetizando o corpo humano (Figura 2(c)). A deformação plástica na plastilina, provocada pelo impacto balístico, permitiu aferir a profundidade de deformação, que foi utilizada como parâmetro para avaliar a capacidade de proteção dos coletes à prova de balas, conforme regulamentado pela NIJ 0101.06 [23]. As profundidades de penetração na plastilina foram medidas por um sensor ótico modelo Q4X da marca Banner.

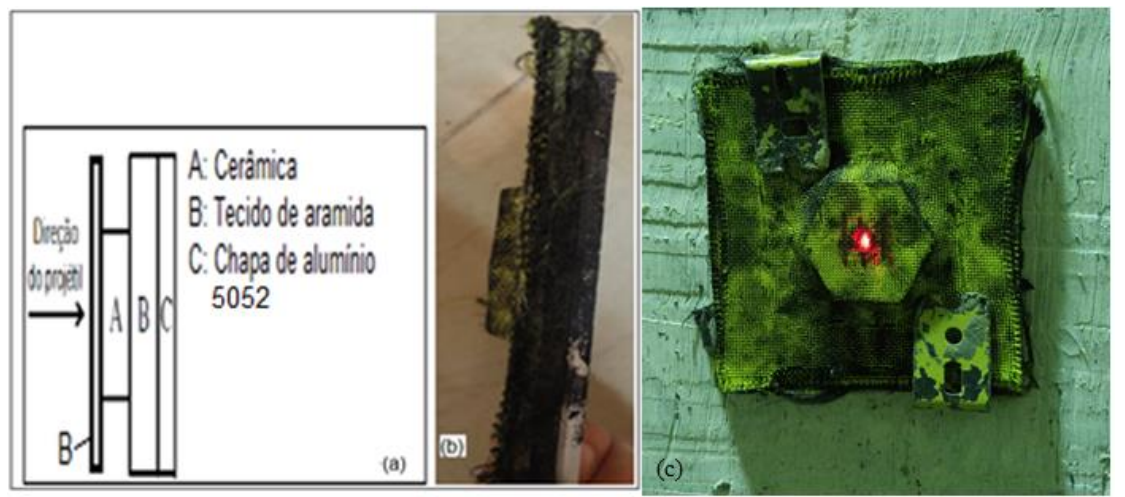

Figura 2. (a) Representação esquemática e (b) exemplar real da blindagem multimacada e (c) sua fixação na plastilina.

Com o objetivo de determinar os valores de pressão e velocidade de partículas nas interfaces entre os materiais envolvidos em impactos balísticos, foi utilizada a técnica de casamento de impedância de choque [19]. Estes parâmetros são obtidos a partir da interseção de curvas características de cada material (curvas Hugoniot), considerando os impactados (alvos), e aqueles que realizam o impacto (projéteis).

A curva Hugoniot característica dos materiais que serão impactados, denominada Hugoniot Direta, é apresentada na Equação 5.

$$
P=\rho_{0}\left(C_{0}+S U_{P}\right) U_{P}
$$

A curva Hugoniot característica dos materiais que realizarão o impacto, denominada Hugoniot invertida, é apresentada na Equação 6.

$$
P=\rho_{0}\left[C_{0}+S\left(V_{i m p}-U_{P}\right)\right]\left(V_{i m p}-U_{P}\right)
$$

Onde $P$ é a pressão, $\rho_{0}$ é a densidade inicial, $C_{0}$ é a velocidade do som, $S$ é uma constante empírica que depende do material, $V_{i m p}$ é a velocidade de impacto e $U_{P}$ é a velocidade da partícula.

\section{RESULTADOS E DISCUSSÃO}

\subsection{Ensaio balístico de velocidade residual}


Os primeiros ensaios balísticos avaliaram o desempenho balístico do composto $\mathrm{Al}_{2} \mathrm{O}_{3}-\mathrm{Nb}_{2} \mathrm{O}_{5}-\mathrm{LiF}$, quanto à absorção de energia, em relação ao composto cerâmico $\mathrm{Al}_{2} \mathrm{O}_{3}-\mathrm{Nb}_{2} \mathrm{O}_{5}$ sem adição de LiF. Os resultados obtidos estão mostrados na Tabela 1. Comparativamente, observou-se que a energia absorvida pelo composto cerâmico sem a adição do fluoreto de lítio à temperatura de $1400^{\circ} \mathrm{C}$ foi semelhante àquela obtida com a adição de $0,5 \%$ de $\mathrm{LiF}$ à temperatura de $1300^{\circ} \mathrm{C}$. Este resultado sugeriu ser possível reduzir a temperatura de sinterização em $100^{\circ} \mathrm{C}$ usando-se a referida adição de fluoreto de lítio em relação à alumina utilizada como blindagem em trabalhos anteriores $[5,7,8,13,14,15]$. Assim, a inclusão do fluoreto de lítio contribuiu para melhorar a absorção de energia do cerâmico.

Tabela 1. Valores médios das velocidades de impacto e residual, e energia absorvida.

\begin{tabular}{|c|c|c|c|c|}
\hline Amostras & $V_{i}(m / s)$ & $V_{R}(m / s)$ & $E_{a b s}(J)$ & $\% E_{a b s}$ \\
\hline $\begin{array}{l}{\left[\mathrm{Al}_{2} \mathrm{O}_{3}-4 \% \mathrm{Nb}_{2} \mathrm{O}_{5}\right]-0,5 \% \mathrm{LiF}} \\
\text { a } 1300^{\circ} \mathrm{C}\end{array}$ & 848,99 & 726,66 & 933,74 & $26,71 \pm 3,40$ \\
\hline $\begin{array}{l}{\left[\mathrm{Al}_{2} \mathrm{O}_{3}-4 \% \mathrm{Nb}_{2} \mathrm{O}_{5}\right]-0,5 \% \mathrm{LiF}} \\
\text { a } 1350^{\circ} \mathrm{C}\end{array}$ & 845,35 & 722,48 & 932,28 & $26,87 \pm 5,07$ \\
\hline $\begin{array}{l}{\left[\mathrm{Al}_{2} \mathrm{O}_{3}-4 \% \mathrm{Nb}_{2} \mathrm{O}_{5}\right]-0,5 \% \mathrm{LiF}} \\
\text { a } 1400^{\circ} \mathrm{C}\end{array}$ & 848,58 & 704,60 & 1077,33 & $30,75 \pm 9,06$ \\
\hline $\begin{array}{l}\mathrm{Al}_{2} \mathrm{O}_{3}-4 \% \mathrm{Nb}_{2} \mathrm{O}_{5} \\
\text { a } 1400^{\circ} \mathrm{C}\end{array}$ & 844,17 & 711,01 & 999,38 & $28,96 \pm 6,54$ \\
\hline
\end{tabular}

A Figura 3 representa o comportamento da absorção de energia, em porcentagem, pelo composto cerâmico em relação à sua densificação e ao seu módulo de elasticidade. Foi possível observar que valores superiores de densificação e de módulo de elasticidade não resultaram necessariamente em maior porcentagem de energia absorvida, evidenciando o papel da porosidade e o da força de ligação como sendo também parâmetros importantes na absorção de energia, conforme observado por Meyers [19].

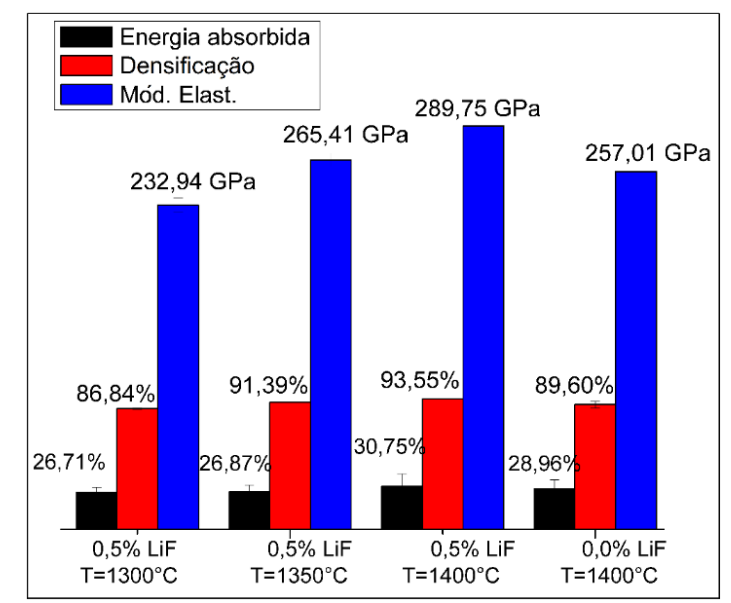

Figura 3. Porcentagem de energia absorvida em relação à densificação e ao módulo de elasticidade.

\subsection{Ensaio balístico de penetração na plastilina}

Na segunda série de ensaios balísticos, o foco foi medir a profundidade de penetração na plastilina. Os resultados obtidos estão apresentados na Tabela 2, e graficamente na Figura 4. De acordo com a norma NIJ [23], uma profundidade de penetração superior a $44 \mathrm{~mm}$ torna-se letal para o ser humano. Todas as amostras com adição 
de LiF sinterizadas nas três temperaturas utilizadas e com a configuração adotada atenderam à exigência da norma, obtendo impressões na plastilina inferiores a $44 \mathrm{~mm}$. Isto confirma a eficiência do novo composto cerâmico estudado e sugerido como novo componente cerâmico em blindagens multicamadas, mesmo sinterizado a temperaturas inferiores, como $1300^{\circ} \mathrm{C}$. Nesta temperatura o custo da confecção da blindagem é reduzido, por ser possível utilizar fornos mais baratos, e de menores custos de manutenção.

Além disso, comparativamente, a impressão deixada na plastilina no composto com $0,5 \%$ de LiF sinterizado a $1300^{\circ} \mathrm{C}$ foi apenas $7 \%$ maior do que aquela produzida no composto sem a adição de LiF sinterizado a $1400^{\circ} \mathrm{C}$. O desempenho da composição cerâmica com LiF sinterizada à baixa temperatura pode estar associado aos valores de dureza obtidos para tais composições, bem como às interações de choque, dependentes da velocidade de onda $\mathrm{C}_{0}$, influenciadas pelo módulo de elasticidade e densidade.

Tabela 2. Valores médios das impressões na plastilina.

\begin{tabular}{|c|c|c|}
\hline Amostras & $V_{i}(m / s)$ & Penetração (mm) \\
\hline $\begin{array}{l}{\left[\mathrm{Al}_{2} \mathrm{O} 3-4 \% \mathrm{Nb}_{2} \mathrm{O}_{5}\right]-0,5 \% \mathrm{LiF}} \\
\text { a } 1300^{\circ} \mathrm{C}\end{array}$ & 846,75 & $37,20 \pm 0,89$ \\
\hline $\begin{array}{l}{\left[\mathrm{Al}_{2} \mathrm{O} 3-4 \% \mathrm{Nb}_{2} \mathrm{O}_{5}\right]-0,5 \% \mathrm{LiF}} \\
\text { a } 1350^{\circ} \mathrm{C}\end{array}$ & 842,72 & $37,44 \pm 1,11$ \\
\hline $\begin{array}{l}{\left[\mathrm{Al}_{2} \mathrm{O}-4 \% \mathrm{Nb}_{2} \mathrm{O}_{5}\right]-0,5 \% \mathrm{LiF}} \\
\text { a } 1400^{\circ} \mathrm{C}\end{array}$ & 848,47 & $34,16 \pm 2,00$ \\
\hline $\mathrm{Al}_{2} \mathrm{O} 3-4 \% \mathrm{Nb}_{2} \mathrm{O}_{5}$ a $1400^{\circ} \mathrm{C}$ & 847,86 & $34,52 \pm 2,23$ \\
\hline
\end{tabular}

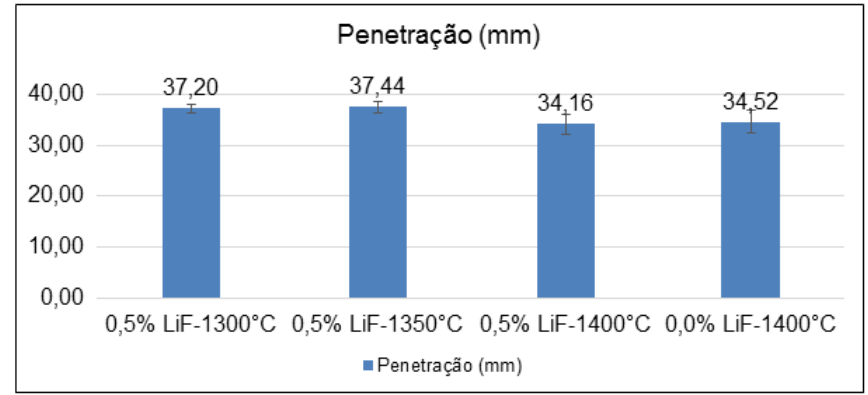

Figura 4. Gráfico da penetração na plastilina.

\subsection{Parâmetros de Choque}

Com base nos resultados obtidos no ensaio balístico de velocidade residual, estimouse os valores de pressão e velocidade de partícula provenientes do impacto do projétil em cada composto cerâmico nos ensaios balísticos (Figura 6). Para isso, utilizou-se a técnica do casamento de impedâncias de choque dos materiais envolvidos..

Para a interação do projétil com o composto aditivado com $0,5 \%$ de LiF, sinterizado à temperatura de $1300^{\circ} \mathrm{C}$, as curvas Hugoniot são mostradas na Figura 6(a), de onde podem ser obtidos graficamente os valores de pressão e de velocidade de partícula. Os valores de $\mathrm{C}_{0}=8,19 \mathrm{~km} / \mathrm{s}$ e $\rho_{0}=3,469 \mathrm{~g} / \mathrm{cm}^{3}$ obtidos experimentalmente na sua condição de processamento foram considerados. $O$ valor empírico do parâmetro $S$ foi aproximado para aquele da $\mathrm{Al}_{2} \mathrm{O}_{3}(\mathrm{~S}=1,299)$, conforme informado por Kleizer [24] (2011), por ter sido a parte majoritária do composto cerâmico, adotado em todos os ensaios balísticos efetuados. Para o projétil à base de chumbo, adotou-se os valores de $\rho_{0}, C_{0}$ e $S$ deste metal, conforme disponibilizado por Meyers [19] (1994). 
Adotou-se a velocidade do projétil como sendo de $848,99 \mathrm{~m} / \mathrm{s}$ conforme obtido pelo radar nos experimentos. A interseção das curvas Hugoniot Direta do alvo cerâmico (Equação 7) e Hugoniot Invertida do projétil (Equação 8) indica velocidade de partícula de $424,36 \mathrm{~m} / \mathrm{s}$, e pressão associada de $12,87 \mathrm{GPa}$ na interface projétil-cerâmico (Figura 5(a)).

$$
\begin{array}{r}
\mathrm{P}_{\text {cer }}=3,469\left(8,19+1,299 \mathrm{U}_{\mathrm{P}}\right) \mathrm{U}_{\mathrm{P}} \\
\mathrm{P}_{\text {roj }}^{\prime}=11,35\left[2,05+1,46\left(0,84899-\mathrm{U}_{\mathrm{P}}\right)\right]\left(0,84899-\mathrm{U}_{\mathrm{P}}\right)
\end{array}
$$
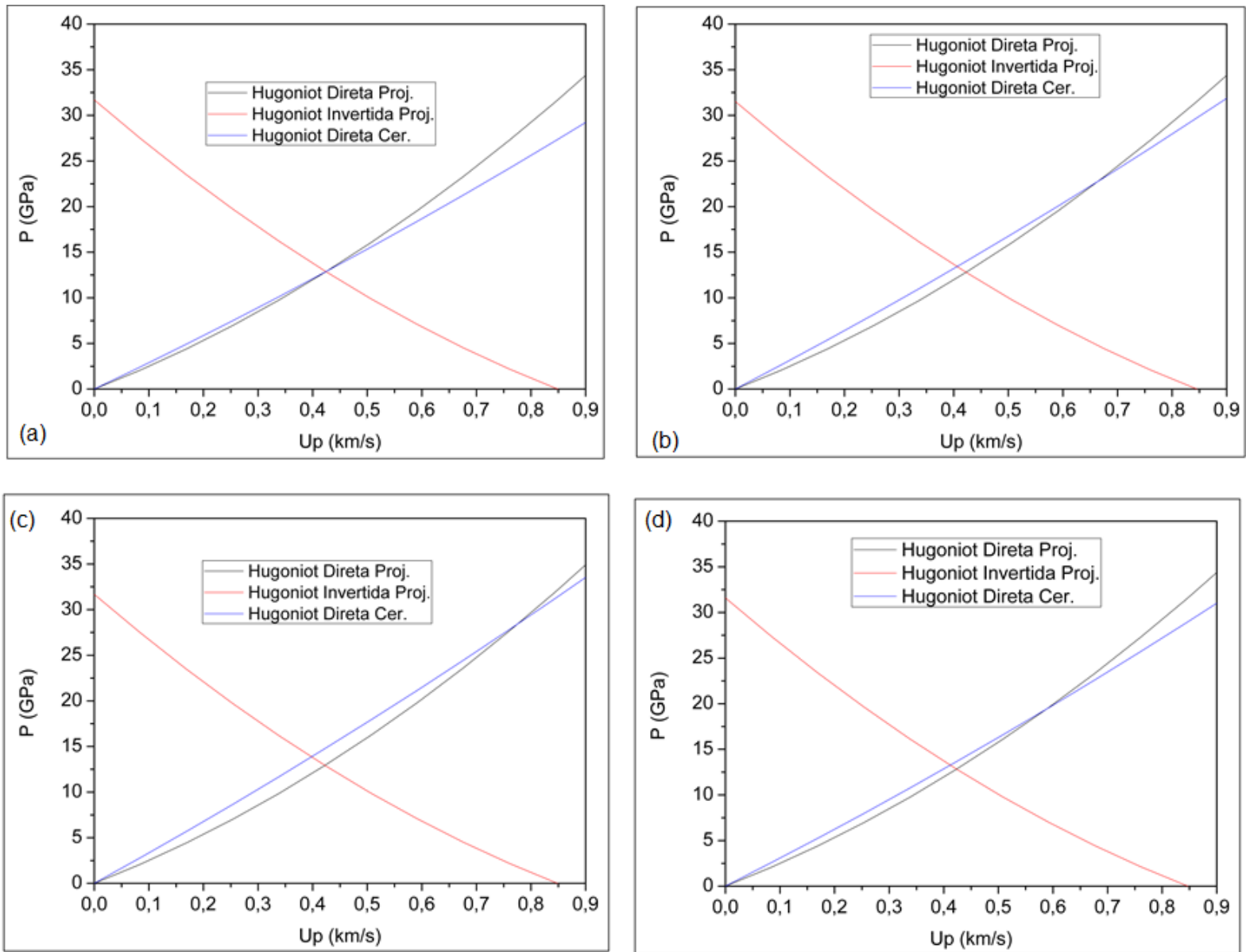

Figura 5 Curvas Hugoniot para a adição de $0,5 \%$ de LiF sinterizada a (a) $1300^{\circ} \mathrm{C}$, (b) $1350^{\circ} \mathrm{C}$, e (c) $1400^{\circ} \mathrm{C}$; e (d) sem a adição de LiF sinterizada a $1400^{\circ} \mathrm{C}$.

As curvas Hugoniot $\mathrm{P} \times \mathrm{Up}$ para a interação do projétil com o composto cerâmico aditivado com $0,5 \%$ de LiF, sinterizado à temperatura de $1350^{\circ} \mathrm{C}$, podem ser observadas na Figura 5(b). Para o cerâmico, os valores de $\mathrm{C}_{0}$ e $\rho_{0}$ foram obtidos experimentalmente neste trabalho com sendo $8,52 \mathrm{~km} / \mathrm{s}$ e $3,653 \mathrm{~g} / \mathrm{cm}^{3}$, respectivamente, considerando-se a velocidade média do projétil de $845,35 \mathrm{~m} / \mathrm{s}$, constante na Tabela 1.

Através da interseção das curvas Hugoniot Direta do alvo cerâmico (Equação 9) e Hugoniot Invertida do projétil (Equação 10), calculou-se os valores de 406,04 m/s para a velocidade de partícula, e de 13,42 GPa de pressão na interface projétil-cerâmico, após igualar as pressões constantes das referidas equações. A Figura 5(b) apresenta este resultado graficamente. 


$$
\begin{array}{r}
\mathrm{P}_{\text {cer }}=3,653\left(8,52+1,299 \mathrm{U}_{\mathrm{P}}\right) \mathrm{U}_{\mathrm{P}} \\
\mathrm{P}_{\text {proj }}^{\prime}=11,35\left[2,05+1,46\left(0,84535-\mathrm{U}_{\mathrm{P}}\right)\right]\left(0,84535-\mathrm{U}_{\mathrm{P}}\right)
\end{array}
$$

Na Figura 5(c) estão ilustradas as curvas Hugoniot para a interação projétil-cerâmico, para a adição de $0,5 \%$ de LiF e temperatura de sinterização de $1400^{\circ} \mathrm{C}$. Os valores de $\mathrm{C}_{0}=8,809 \mathrm{~km} / \mathrm{s}$ e $\rho_{0}=3,7349 \mathrm{~g} / \mathrm{cm}^{3}$ obtidos experimentalmente na sua condição de processamento foram considerados. Adotou-se a velocidade do projétil como sendo de $848,58 \mathrm{~m} / \mathrm{s}$ conforme obtido pelo radar nos experimentos. Ao se igualar a Equação 11, referente à Hugoniot Direta do alvo cerâmico, e Equação 12, referente à Hugoniot Invertida do projétil, foram obtidos valores de 13,85 GPa de pressão e de $397,85 \mathrm{~m} / \mathrm{s}$ de velocidade de partícula.

$$
\begin{array}{r}
\mathrm{P}_{\text {cer }}=3,734\left(8,809+1,299 \mathrm{U}_{\mathrm{P}}\right) \mathrm{U}_{\mathrm{P}} \\
\mathrm{P}_{\text {proj }}^{\prime}=11,35\left[2,05+1,46\left(0,84858-\mathrm{U}_{\mathrm{P}}\right)\right]\left(0,84858-\mathrm{U}_{\mathrm{P}}\right)
\end{array}
$$

As curvas Hugionot do alvo cerâmico sem adição de fluoreto de lítio, ou seja, composto apenas de $\mathrm{Al}_{2} \mathrm{O}_{3}-4 \% \mathrm{Nb}_{2} \mathrm{O}_{5}$, e do projétil, estão dispostas na Figura $6(\mathrm{~d})$. A Equação 13 apresenta a Hugoniot Direta deste cerâmico, com $\mathrm{C}_{0}=8,474 \mathrm{~km} / \mathrm{s}, \rho_{0}$ $=3,573 \mathrm{~g} / \mathrm{cm}^{3}$, enquanto a Equação 14 apresenta a Hugoniot Invertida do projétil, considerando sua velocidade como $846,92 \mathrm{~m} / \mathrm{s}$. Essa interação gerou uma pressão de 13,26 GPa na interface projétil-cerâmico, com velocidade de partícula de 411,87 $\mathrm{m} / \mathrm{s}$, com a solução gráfica mostrada na Figura 5(d).

$$
\begin{array}{r}
\mathrm{P}_{\text {cer }}=3,573\left(8,474+1,299 \mathrm{U}_{\mathrm{P}}\right) \mathrm{U}_{\mathrm{P}} \\
\mathrm{P}_{\text {proj }}^{\prime}=11,35\left[2,05+1,46\left(1,69798-\mathrm{U}_{\mathrm{P}}\right)\right]\left(1,69384-\mathrm{U}_{\mathrm{P}}\right)
\end{array}
$$

Foi possível observar que o valor de pressão gerado pelo impacto do projétil no alvo de composto cerâmico $\left[\mathrm{Al}_{2} \mathrm{O}_{3}-4 \% \mathrm{Nb}_{2} \mathrm{O}_{5}\right]-0,5 \% \mathrm{LiF}$, sinterizado a $1300^{\circ} \mathrm{C}$, foi de $12,87 \mathrm{GPa}$, enquanto que para o composto $96 \% \mathrm{Al}_{2} \mathrm{O}_{3}-4 \% \mathrm{Nb}_{2} \mathrm{O}_{5}$ sinterizado a $1400^{\circ} \mathrm{C}$, utilizado em trabalhos anteriores, a pressão foi de 13,26 GPa. Ou seja, houve uma diferença relativa de aproximadamente $3 \%$. Portanto, pode-se considerar que a pressão gerada foi aproximadamente a mesma para as amostras.

Este fato reforça o indício de uma eficiência comparável entre o composto cerâmico desenvolvido neste trabalho com aquele desenvolvido por Gomes [5], apresentando uma redução na temperatura de sinterização, considerável, de $100^{\circ} \mathrm{C}$. Isto representa uma redução no custo da produção desta blindagem. Na Tabela 3 estão contidos os valores de pressão e de velocidade de partícula estimados pela técnica de casamento de impedâncias de choque.

Tabela 3. Valores de pressão e de velocidade de partículaestimados pela técnica de casamento de impedâncias de choque.

\begin{tabular}{lcc}
\hline Amostras & $\mathbf{P}$ (GPa) & $\mathbf{U}_{\mathbf{P}}(\mathbf{k m} / \mathbf{s})$ \\
\hline$\left[\mathrm{Al}_{2} \mathrm{O} 3-4 \% \mathrm{Nb}_{2} \mathrm{O}_{5}\right]-0,5 \% \mathrm{LiF}$ a $1300^{\circ} \mathrm{C}$ & 12,87 & 424,36 \\
\hline$\left[\mathrm{Al}_{2} \mathrm{O}-4 \% \mathrm{Nb}_{2} \mathrm{O}_{5}\right]-0,5 \% \mathrm{LiF}$ a $1350^{\circ} \mathrm{C}$ & 13,42 & 406,64 \\
\hline$\left[\mathrm{Al}_{2} \mathrm{O} 3-4 \% \mathrm{Nb}_{2} \mathrm{O}_{5}\right]-0,5 \% \mathrm{LiF}$ a $1400^{\circ} \mathrm{C}$ & 13,85 & 397,85 \\
\hline $\mathrm{Al}_{2} \mathrm{O} 3-4 \% \mathrm{Nb}_{2} \mathrm{O}_{5}$ a $1400^{\circ} \mathrm{C}$ & 13,26 & 411,87 \\
\hline
\end{tabular}


Assim, de acordo com os resultados obtidos, a adição de LiF, mais especificamente a fração de $0,5 \%$ em peso, possibilitou a redução da temperatura de sinterização para $1300^{\circ} \mathrm{C}$ sem perdas significativas de suas propriedades.

\section{CONCLUSÕES}

Neste trabalho, um novo sistema cerâmico ainda não estudado teve suas propriedades elásticas investigadas. Além disso, seus parâmetros de choque, de significância para potencial aplicação balística, também foram levantados. Dessa forma, pode-se concluir que:

1) A adição de fluoreto de lítio ao sistema alumina-nióbia foi benéfica para as propriedades elásticas, comparadas à mesma temperatura.

2) Os resultados obtidos das propriedades elásticas da composição cerâmica com LiF sinterizada em diferentes temperaturas revelaram ser possível reduzir em até $100^{\circ} \mathrm{C}$ a temperatura de sinterização, sem haver redução significativa nas propriedades elásticas obtidas em relação às composições cerâmicas de trabalhos anteriores [5,7]. 3) Os valores de pressão $(P)$ e de velocidades de partícula $\left(U_{P}\right)$ na interface de impacto projétil-cerâmica revelaram valores bem próximos entre o sistema alumina-nióbia e o sistema alumina-nióbia-fluoreto de lítio, com a composição utilizada neste trabalho, em temperatura de sinterização $100^{\circ} \mathrm{C}$ inferior.

4) Como esperado, os maiores valores de pressão na interface de impacto foram obtidos pelas amostras com os maiores valores de módulo de elasticidade e de velocidade de onda elástica.

5) Pela representativa redução na temperatura de sinterização, há uma significativa redução no custo de processamento do material apresentado neste trabalho, que apresentou interessantes resultados para potencial aplicação balística, como em sistemas de blindagem multicamada.

\section{Agradecimentos}

Os autores gostariam de agradecer à CAPES pelo financiamento, e ao CAEx (Centro de Avaliações do Exército) pelos ensaios balísticos.

\section{REFERÊNCIAS}

1 Medvedovski E. Ballistic Performance of Armour Ceramics: Influence of Design and Structure-Part 1. Ceramics International. 2010; 36: 2103-2115.

2 Silva MV, Stainer D, AI-Qureshi HA, Hotza D. Blindagens Cerâmicas para Aplicações Balísticas: uma revisão. Cerâmica. 2014; 60: 323-331.

3 James B. Practical issues in ceramic armor design. Ceramic Armor Material by Design. 2002; 134: 23-31.

4 Yadav S, Ravichandran G. Penetration Resistance of Laminated Ceramic/Polymer Structures. International Journal of Impact Engineering. 2003; 28: 557-574.

5 Gomes AV. Comportamento balística da alumina com adição de nióbia e variação da geometria do alvo. Tese de doutorado - IME. Rio de Janeiro. 2004.

6 Medvedovski E. Lightweight Ceramic Composite Armour System.Advances in Applied Ceramics. 2006; 105: 241-245.

7 Trindade W. Influência da Geometria e da Microestrutura no Comportamento Dinâmico da Alumina Adititivada com Nióbia. Tese de doutorado - IME. Rio de Janeiro, 2012.

8 Chagas CFMC. Minimização do Efeito de Aresta em Blindagem Cerâmica à Base de Alumina. Tese de doutorado - IME. Rio de Janeiro, 2014. 
9 Monteiro SN, Lima Jr EP, Louro LHL, Da Silva LC, Drelich JW. Unlocking Function of Aramid Fibers in Multilayered Ballistic Armor. Metallurgical and Materials Transactions A. 2014; 46A: 37-40.

10 Serjouei A, Chi R, Zhang Z, Sridhar I. Experimental Validation of BLV Model on Bi-Layer Ceramic-Metal Armor. International Journal of Impact Engineering. 2015; 77: 30-41.

11 Jesus PRR. Efeito da adição do fluoreto de lítio na densificação da alumina aditivada com 4\% em peso de nióbia. Dissertação de mestrado - IME. Rio de Janeiro, 2016.

12 Santos JL. Comportamento balístico de componente cerâmico à base de $\mathrm{Al}_{2} \mathrm{O}_{3}-\mathrm{Nb}_{2} \mathrm{O}_{5}-$ LiF em blindagem multicamada. Tese de doutorado - IME. Rio de Janeiro, 2016.

13 Candido VS. Caracterização e propriedades de compósitos poliméricos reforçados com fibras de bagaço de cana-de-açúcar. Tese de doutorado - IME. Rio de Janeiro, 2014.

14 Braga FO. Comportamento Balístico de uma Blindagem Multicamada utilizando Compósito Poliéster-Curauá como Camada Intermediária. Dissertação de mestrado IME. Rio de Janeiro, 2015.

15 Da Luz FS. Avaliação do Comportamento Balístico de Blindagem Multicamada do Compósito de Epóxi reforçado com Fibra de Juta. Dissertação de mestrado - IME. Rio de Janeiro, 2014.

16 De Araújo BM. Avaliação do Comportamento Balístico de Blindagem Multicamada com Compósito de Epóxi reforçado com Fibra de Sisal. Dissertação de mestrado - IME. Rio de Janeiro, 2015.

17 Da Cruz RB. Avaliação do Comportamento Balístico de Blindagem Multicamadas com Compósitos de Epóxi reforçados por Fibras de Bambu.

18 Milanezi TL. Avaliação do Comportamento Sinergético de Blindagem Multicamadas com a Fibra de Rami. Dissertação de mestrado - IME. Rio de Janeiro, 2015.

19 Meyers MA. Dynamic Behavior of Materials. New York: John Wiley \& Sons, 1994.

20 Rankine WJM. On the thermodynamic theory of waves of finite longitudinal disturbance. Philosophical Transactions of the Royal Society of London. 1870: 160; 277-286.

21 Hugoniot $\mathrm{PH}$. Mémoire sur la propagation du mouvement dans les corps et plus spécialement dans les gaz parfaits. 2e Partie. J. Ecole Polytech. 1889: 58; 1-125.

22 ABNT NBR 15000. Blindagens para impactos balísticos - Classificação e critérios de avaliação. 2005.

23 NIJ Standard 0101.06. Ballistic Resistance of Personal Body Armor. U.S. Department of Justice/Office of Justice Programs. 2008.

24 Kleiser GJ, Chhabildas LC, Reinhart WD. Comparison of Dynamic Compression Behavior of Single Crystal Sapphire to Polycrystalline Alumina. International Journal of Impact Engineering. 2011: 38; 473-479. 